\title{
Cardiovascular risk factors and major depressive disorder: a cross-sectional study in São Paulo, Brazil
}

\author{
Danielle Bivanco-Lima', Itamar de Souza Santos"', Yuan-Pang Wang'", Maria Carmen Vianal', Laura Helena Andradev,

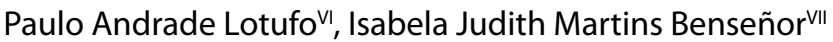 \\ Section of Psychiatric Epidemiology (LIM-23), Institute of Psychiatry, Faculdade de Medicina FMUSP, Universidade de Sao Paulo, Sao Paulo, SP, BR
}

'MD, PhD. Professor, Department of Public Health, Faculdade de Ciências Médicas da Santa Casa de São Paulo (FCMSP), São Paulo (SP), Brazil. (D) orcid.org/0000-0001-9243-0232

"MD, PhD. Center for Clinical and

Epidemiological Research, Hospital Universitário (HU), Universidade de Sao Paulo, Sao Paulo, $\mathrm{SP}, \mathrm{BR}$, and Professor, Department of Internal Medicine, Faculdade de Medicina FMUSP, Universidade de Sao Paulo, Sao Paulo, SP, BR. (D) orcid.org/0000-0003-3212-8466

'"MD, PhD. Assistant Professor, Section of Psychiatric Epidemiology (LIM-23), Institute of Psychiatry, Hospital das Clínicas FMUSP, Faculdade de Medicina, Universidade de São Paulo, Sao Paulo, SP, BR.

(D) orcid.org/0000-0001-7076-8312

"MD, PhD. Professor, Postgraduate Program on Collective Health, Universidade Federal do Espírito Santo (UFES), Vitória (ES), Brazil.

(D) orcid.org/0000-0002-0464-4845

vMD, PhD. Assistant Professor, Section of Psychiatric Epidemiology (LIM-23), Institute of Psychiatry, Hospital das Clínicas FMUSP, Faculdade de Medicina, Universidade de São Paulo, Sao Paulo, SP, BR.

(D) orcid.org/0000-0002-2362-3521

vMD, DrPH. Coordinator, Center for Clinical and Epidemiological Research, Hospital Universitário (HU), Universidade de Sao Paulo, Sao Paulo, SP, $\mathrm{BR}$, and Full Professor, Department of Internal Medicine, Faculdade de Medicina FMUSP, Universidade de Sao Paulo, Sao Paulo, SP, BR. (D) orcid.org/0000-0002-4856-8450

VIIMD, PhD. Deputy Coordinator, Center for Clinical and Epidemiological Research, Hospital Universitário (HU), Universidade de Sao Paulo, Sao Paulo, SP, BR, and Full Professor, Department of Internal Medicine, Faculdade de Medicina FMUSP, Universidade de Sao Paulo, Sao Paulo, SP, BR.

(D) orcid.org/0000-0002-6723-5678

\section{KEY WORDS (MeSH terms):}

Depression.

Risk factors.

Cardiovascular diseases.

Depressive disorder, major.

Depressive disorders.

\section{AUTHORS' KEY WORDS:}

Cardiovascular risk.

Major depressive disorder.

Depressed individuals.

Lifestyle and risk factors.

\begin{abstract}
BACKGROUND: Cardiovascular risk factors can mediate the association between depression and cardiovascular diseases.

OBJECTIVE: To evaluate cardiovascular risk factors in adult individuals with and without histories of major depression in the metropolitan region of São Paulo, Brazil.

DESIGN AND SETTING: Cross-sectional study in São Paulo (SP), Brazil.

METHODS: This study evaluated 423 individuals without any lifetime diagnosis of major depression and 203 individuals with a previous diagnosis of major depression $(n=626)$. The participants underwent a psychiatric evaluation using a structured clinical interview (SCID-1), an anthropometric evaluation and a clinical evaluation that included blood pressure measurement and assessment of fasting blood glucose, lipid profile and physical activity levels.

RESULTS: Individuals with histories of major depression were more likely to be female $(P<0.0001)$. Individuals with lifetime diagnoses of major depression were more likely to be current smokers (odds ratio, $\mathrm{OR}$ 1.61; $95 \%$ confidence interval, $\mathrm{Cl} 1.01-2.59$ ) and to have diabetes (OR 1.79; 95\% Cl 1.01-3.21); and less likely to be obese (OR 0.58; 95\% Cl 0.35-0.94).

CONCLUSION: Individuals with major depression had higher odds of presenting tobacco smoking and diabetes, and lower odds of being obese. Healthcare professionals need to be aware of this, so as to increase the rates of diagnosis and treatment in this population.
\end{abstract}

\section{INTRODUCTION}

Cardiovascular diseases are the leading cause of death worldwide and in Brazil. ${ }^{1}$ Mortality rates relating to cardiovascular diseases have also been found to be higher in a psychiatric population than in an age-matched general population, with a hazard ratio (HR) of 4.16 (95\% confidence interval, CI 1.22-14.25). ${ }^{2}$ There is reasonable data describing the association between depressive disorders and cardiovascular disease incidence and mortality, especially with regard to coronary heart diseases. ${ }^{3,4}$ Nicholson et al. reviewed 54 observational studies and conducted a meta-analysis regarding depression as an etiological factor among individuals with coronary heart diseases. They found that depression increased the risk of incident coronary heart disease (pooled relative risk, RR 1.81; 95\% CI 1.53-2.15). ${ }^{5}$

Individuals' profile of cardiovascular risk factors can explain, at least partially, the higher incidence of coronary heart disease and related mortality among individuals with depressive disorders. Appleton et al. evaluated 8,138 men and found higher incidence of cardiovascular diseases in individuals with depressive disorders (HR 2.36; 95\% CI 1.39-4.0). However, the association lost significance after cardiovascular risk factors were included in the model for adjustment (HR 1.73; 95\% CI 0.99-2.99). ${ }^{2}$ Therefore, cardiovascular risk factors might be important mediators of the causal model linking depressive disorders and cardiovascular mortality.

Depressed individuals might have unhealthier lifestyles than the general population, with higher rates of smoking and greater physical inactivity. ${ }^{6,7}$ Strine et al. evaluated 217,379 individuals in a survey in the United States and found that individuals with previous major depression had higher odds of current smoking (odds ratio, OR 1.9; 95\% CI 1.8-2.1) and physical inactivity (OR 1.3; 95\% CI 1.2-1.4). ${ }^{7}$ There are also data showing higher prevalence of metabolic syndrome in patients with major depression ${ }^{8}$ and, more consistently, in depressed women. ${ }^{9,10}$ 
Studies that have evaluated the relationship between cardiovascular risk factors and depressive disorders in the general population are less common, especially in low and middle-income countries.

\section{OBJECTIVE}

The aim of this study was to evaluate cardiovascular risk factors (hypertension, diabetes, dyslipidemia, smoking, obesity and metabolic syndrome) and their association with lifetime major depression in a subsample of individuals from the São Paulo Megacity Mental Health Survey, in the metropolitan area of São Paulo, Brazil.

\section{METHODS}

\section{Study design}

The São Paulo Megacity Mental Health Survey is a populationbased cross-sectional study with the aim of evaluating the prevalence of psychiatric morbidity. It focuses on non-institutionalized Portuguese-speaking adults aged 18 or older, living in the metropolitan area of São Paulo, state of São Paulo, Brazil, and it forms the Brazilian component of the World Mental Health Survey (WMH). This is an initiative from the World Health Organization (WHO) that comprises a series of population-based epidemiological studies of adult resident populations in over 30 participating countries around the world. It is conducted with the same methodological framework, using the same instruments as in the WMH. The study protocol has been detailed elsewhere. ${ }^{11}$

\section{Study sample}

The current study was based on a subsample of the Brazilian $\mathrm{WMH}$, with a cross-sectional design and was conducted in parallel with the main household survey. Out of the 5,037 individuals who were evaluated in their households, a random subsample of 780 individuals was selected. Eight individuals (1\%) with symptoms that needed emergency evaluation, who did not complete the protocol, were excluded. We also excluded 146 (18.7\%) who had substance use disorders or eating disorders and, thus, 626 individuals (80.3\%) remained included in the final analysis (Figure 1). The only missing data consisted of body mass index (BMI) values for individuals. The remainder of the data was $100 \%$ complete.

\section{Psychiatric and clinical assessments}

In the hospital outpatient setting, trained psychiatrists assessed participants through the Structured Clinical Interview for DSM-IV Axis I Disorders (SCID-I). ${ }^{12}$ The SCID-I makes it possible to generate current and lifetime diagnoses on psychiatric conditions. For this analysis, we only included individuals with lifetime diagnoses of major depressive disorder (MDD), with or without associated anxiety disorders.

In addition, the onsite evaluation included a questionnaire that was used to collect the following sociodemographic data: age, sex, marital status, number of years of formal education (measured as number of school years), current job status (worker, housekeeper, student, retired or other) and annual household income. A clinical evaluation focusing on cardiovascular risk factors and cardiovascular diseases was performed by two physicians. All subjects were asked about smoking status (never, past or current), alcohol intake and personal histories of hypertension, diabetes, coronary heart disease or cerebrovascular disease. The level of physical activity was measured using the long version of the International Physical Activity Questionnaire (IPAQ) ${ }^{13}$ Inadequate physical activity was defined as being sedentary or insufficiently active according to the IPAQ, considering two domains separately: leisure-time and journey-to-work (commuting) physical activity.

\section{Definition of cardiovascular risk factors}

Hypertension was defined as mean systolic blood pressure $\geq 140$ $\mathrm{mmHg}$, mean diastolic blood pressure $\geq 90 \mathrm{mmHg}$, medical history of hypertension and/or current use of medication to treat hypertension. ${ }^{14}$ Diabetes was defined as fasting blood glucose $\geq$ $126 \mathrm{mg} / \mathrm{dl}$, medical history of diabetes and/or use of medication to treat diabetes. ${ }^{15}$

Individuals were considered to have dyslipidemia if they had one or more lipid profile alterations: high-density lipoprotein-cholesterol (HDL-c) levels lower than $40 \mathrm{mg} / \mathrm{dl}$ for men and lower than $50 \mathrm{mg} / \mathrm{dl}$ for women; triglyceride (TG) levels higher than $150 \mathrm{mg} / \mathrm{dl}$; or low-density lipoprotein-cholesterol (LDL-c) levels higher than $130 \mathrm{mg} / \mathrm{dl}$. Metabolic syndrome was defined as the presence of three or more of the following criteria: abdominal obesity (waist circumference $\geq 102$ centimeters $(\mathrm{cm})$ in men and $\geq 88$ $\mathrm{cm}$ in women); TG blood level $\geq 150 \mathrm{mg} / \mathrm{dl}$; HDL-c cholesterol blood levels lower than $40 \mathrm{mg} / \mathrm{dl}$ in men or lower than $50 \mathrm{mg} / \mathrm{dl}$

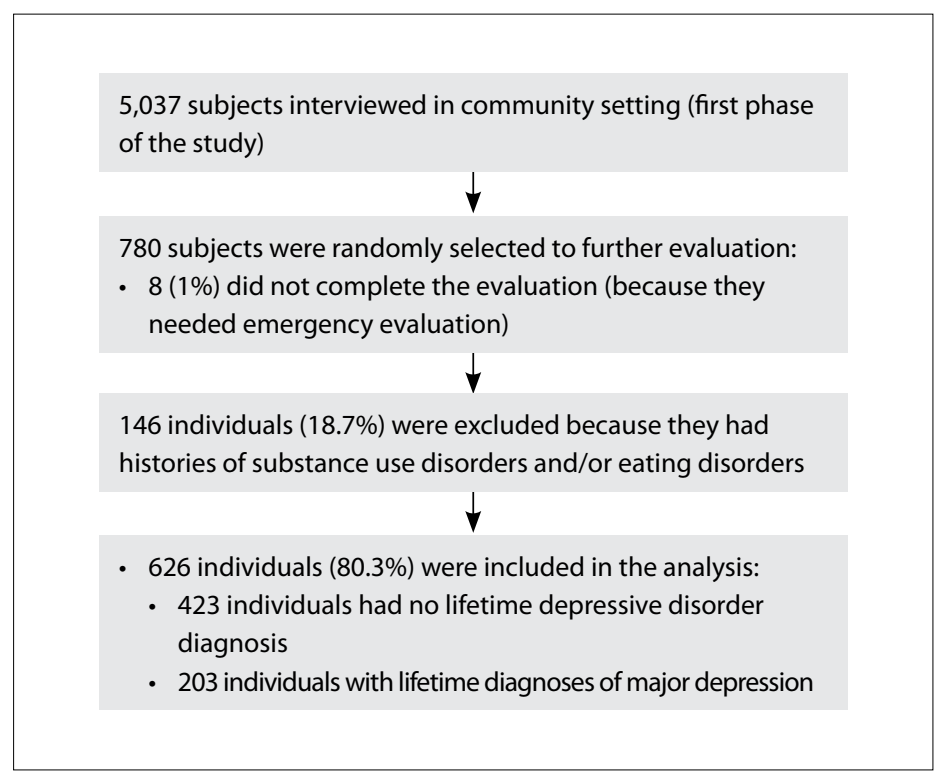

Figure 1. Subjects included in the second phase of the São Paulo Megacity Survey. 
in women; blood pressure $(\mathrm{BP}) \geq 130 \mathrm{mmHg}$ of systolic blood pressure and/or $\geq 85 \mathrm{mmHg}$ of diastolic blood pressure; or fasting glucose blood level $\geq 100 \mathrm{mg} / \mathrm{dl} .{ }^{16}$

\section{Anthropometric and blood pressure measurements}

The anthropometric evaluation (weight, height and waist circumference) was performed using standardized equipment and techniques. ${ }^{17}$ Blood pressure was measured three times using a standardized technique in the seated position after a five-minute rest. Body mass index (BMI) was calculated as weight in kilograms divided by height in meters squared. ${ }^{18}$ Overweight was defined as BMI between 25 and $29.9 \mathrm{~kg} / \mathrm{m}^{2}$. Obesity was defined as BMI greater than or equal to $30 \mathrm{~kg} / \mathrm{m}^{2}$.

\section{Laboratory measurements}

Venous blood samples were obtained after a 12-hour overnight fast. The blood samples were analyzed for the following: glucose levels (hexokinase method); total cholesterol (TC) (enzymatic colorimetric assay); HDL-c (HDL - homogeneous cholesterol); triglycerides (enzymatic colorimetric assay); high-sensitivity C-reactive protein (nephelometry); thyroid-stimulating hormone (TSH); and free thyroxine (immunoenzymatic assay - third generation). LDL-c values were obtained using the Friedewald formula. ${ }^{16}$

\section{Statistical analysis}

Data entry was carried out twice and a validity check was done in order to identify and correct any data entry errors. Groups were compared using the chi-square test or Fisher's exact test for categorical data, whenever applicable. Continuous variables were tested for normality, and one-way ANOVA (analysis of variance) or nonparametric tests were performed, whenever applicable. We used logistic regression to evaluate comparisons among individuals with and without depression and the results were presented crudely and adjusted for age and sex. The significance level was set at 0.05 . We used the SPSS for Windows software, version 16.0 (SPSS Inc., Chicago, United States), and the R software, version 3.0.0 (Vienna, Austria) for the analyses.

\section{Ethical considerations}

The Institutional Review Board of Hospital das Clínicas, Universidade de São Paulo approved the study under the protocol number 234/03 (on April 24, 2003), and all the participants signed a written informed consent statement.

\section{RESULTS}

Among the 626 participants, 383 (61.2\%) were female, 423 had no lifetime history of MDD (67.6\%; 95\% CI 63.8-71.2) and 203 had lifetime histories of MDD (32.4\%; 95\% CI 28.9-36.2). The mean age was 40.5 years among the individuals without any lifetime diagnosis of major depressive disorder and 41.5 years among the individuals with lifetime histories of major depressive disorder $(\mathrm{P}$ $=0.24)$. A total of 423 individuals (67.6\%) had no lifetime history of depressive disorder, while 203 (32.4\%) had a SCID-I diagnosis of lifetime depressive disorder. Individuals with lifetime depression were more likely to be current smokers than were individuals who had never been depressed, with borderline significance (20.3\% versus $13.0 \% ; \mathrm{P}=0.056$ ) (Table $\mathbf{1}$ ).

There were no differences between the individuals with and without lifetime diagnoses of major depressive disorder regarding cardiovascular risk factors, except for a borderline difference for current tobacco smoking (13.0\% among individuals without MDD and $20.2 \%$ among individuals with MDD; $\mathrm{P}=0.056$ ). Most of the individuals were obese or overweight (64.5\% of the individuals without MDD and $61.0 \%$ of the individuals with MDD; $\mathrm{P}=0.12$ ). High frequencies of hypertension ( $37 \%$ in both groups; $\mathrm{P}=0.99$ ), metabolic syndrome (34.8\% versus $33.5 \%$ in individuals without and with MDD, respectively; $\mathrm{P}=0.79$ ) and dyslipidemia ( $62.4 \%$ of individuals without MDD and 55.2\% of individuals with MDD; $\mathrm{P}=0.10$ ) were observed. There was high frequency of sedentary behavior, particularly with regard to physical activity during leisure time (Table 1).

In the logistic regression, crude analysis showed that individuals with lifetime histories of MDD had odds ratios for body mass index, hypertension, diabetes mellitus, dyslipidemia, metabolic syndrome and leisure-time physical activity that were similar to those of individuals without MDD. Also in the crude analysis, individuals with MDD had higher odds ratios for current smoking (OR 1.65; 95\% CI 1.04-2.62) and lower odds of active commuting-related physical activity (OR 0.60; 95\% CI 0.39-0.93) (Table 2).

In the age and sex-adjusted logistic regression model, individuals with lifetime major depression had higher odds of being current smokers (OR 1.61; 95\% CI 1.01-2.59) and higher odds of presenting diabetes (OR 1.79; 95\% CI 1.01-3.21). In addition, individuals with lifetime major depression had lower odds of presenting obesity (OR 0.58; 95\% CI 0.35-0.94). No differences were found for other cardiovascular risk factors (hypertension, dyslipidemia, metabolic syndrome and physical activity) (Table 2). Further adjustment for cardiovascular risk factors did not change the results (data not shown).

\section{DISCUSSION}

In our study, we found that current smoking and diabetes showed associations with lifetime histories of major depression. We also found lower odds of being obese among individuals with lifetime histories of major depression, compared with nondepressed individuals.

Our study found that individuals with lifetime major depression had higher odds of being diabetic. Most studies have found this same association between diabetes and major depression, including evidence for a bidirectional association..$^{19,20-23}$ Mommersteeg et al. found among 231,797 individuals from 47 countries that diabetes increased the odds 
of depressive symptoms (OR 2.36; 95\% CI 1.91-2.92). ${ }^{24}$ Mezuk et al. found that diabetes could enhance the risk of incident depression (OR 1.15; 95\% CI 1.02-1.30). They also found higher risk of new-onset diabetes among depressed subjects (RR 1.60; 95\% CI 1.37-1.88). ${ }^{23}$

There are multiple explanations for the association between diabetes and depression, as mediated by long-term use of antidepressants $^{25}$ and increased inflammation and insulin resistance. ${ }^{26}$ The recent discussion on syndemics has indicated that the association between diabetes and depression is more complex than it would seem through explanations that invoke exclusive biological pathways. The dynamics of poverty and sociocultural contexts influence lifestyle and the health and disease process that links these two diseases. ${ }^{27-30}$ Because the present study was a cross-sectional analysis, it is impossible to draw any conclusions relating to causality. Nevertheless, our data confirm the presence of this association in a Brazilian population sample.
We also found higher odds of current tobacco use among individuals with lifetimes of depressive disorder. Other studies have reported similar results. ${ }^{7,31}$ Strine et al. found higher odds of tobacco use among currently depressed subjects (OR 2.2; 95\% CI 2.0-2.3) and previously depressed subjects (OR 1.9; 95\% CI 1.82.1). ${ }^{7}$ Goodwin et al. found that among smokers the prevalence of past-year depression was $10.45 \%$, compared with $5.51 \%$ among never-smokers. ${ }^{32}$ Previous Brazilian studies confirmed the association between tobacco smoking and depressive symptoms. ${ }^{33,34}$ Borçói et al. found a prevalence ratio of 2.69 (95\% CI 1.62-4.46) for being a current smoker, among individuals with depressive disorder in a sample at a primary care service in a Brazilian municipality (Alegre, Espírito Santo). ${ }^{33}$ Rocha et al. evaluated 1,054 individuals in a population-based survey in a southern municipality (Florianópolis, Santa Catarina) and found that $29 \%$ of current smokers had depressive symptoms. ${ }^{34}$

Table 1. Cardiovascular disease risk factors according to the presence of lifetime histories of major depressive disorder (MDD)

\begin{tabular}{|c|c|c|c|c|c|}
\hline & \multicolumn{4}{|c|}{ Lifetime history of major depressive disorder } & \multirow{3}{*}{$\mathbf{P}$} \\
\hline & \multicolumn{2}{|c|}{ No $(n=423)$} & \multicolumn{2}{|c|}{ Yes $(n=203)$} & \\
\hline & $\mathbf{N}$ & $\%(95 \% \mathrm{Cl})$ & $\mathbf{N}$ & $\%(95 \% \mathrm{Cl})$ & \\
\hline Sex & & & & & $<0.001$ \\
\hline Female & 228 & $53.9(49.1-58.6)$ & 155 & $76.4(70.1-81.8)$ & \\
\hline Male & 195 & $46.1(41.4-50.9)$ & 48 & $23.6(18.2-29.9)$ & \\
\hline Mean age in years (SD) & $40.5(11.1)$ & $40.5(39.4-41.6)$ & $41.5(10.6)$ & $41.5(40.0-43.0)$ & 0.243 \\
\hline Smoking status & & & & & 0.056 \\
\hline Never & 257 & $60.8(56.0-65.3)$ & 116 & $57.1(50.3-63.8)$ & \\
\hline Past & 110 & $26.0(22.0-30.4)$ & 45 & $22.2(16.9-28.3)$ & \\
\hline Current & 55 & $13.0(10.1-16.5)$ & 41 & $20.2(15.1-26.1)$ & \\
\hline Body mass index & & & & & 0.118 \\
\hline Normal $<25.0 \mathrm{~kg} / \mathrm{m}^{2}$ & 149 & $35.2(30.8-39.9)$ & 78 & $38.4(31.9-45.3)$ & \\
\hline Overweight: $25.0-29.9 \mathrm{~kg} / \mathrm{m}^{2}$ & 164 & $38.8(34.2-43.5)$ & 86 & $42.4(35.7-49.3)$ & \\
\hline Obese $\geq 30.0 \mathrm{~kg} / \mathrm{m}^{2}$ & 107 & $25.3(21.3-29.6)$ & 36 & $17.7(12.9-23.5)$ & \\
\hline Hypertension & & & & & 0.990 \\
\hline No & 265 & $62.6(58.0-67.2)$ & 128 & $63.1(56.3-69.5)$ & \\
\hline Yes & 158 & $37.4(32.8-42.0)$ & 75 & $36.9(30.5-43.8)$ & \\
\hline Diabetes mellitus & & & & & 0.105 \\
\hline No & 390 & $92.9(89.3-94.5)$ & 179 & $88.2(83.2-92.1)$ & \\
\hline Yes & 33 & $7.8(5.5-10.7)$ & 24 & $11.8(7.9-16.8)$ & \\
\hline Dyslipidemia & & & & & 0.098 \\
\hline No & 159 & $37.6(33.1-42.3)$ & 91 & $44.8(38.1-51.7)$ & \\
\hline Yes & 264 & $62.4(57.7-66.9)$ & 112 & $55.2(48.3-61.9)$ & \\
\hline Metabolic syndrome & & & & & 0.788 \\
\hline No & 276 & $65.2(60.6-69.7)$ & 135 & $66.5(59.8-72.7)$ & \\
\hline Yes & 147 & $34.8(30.3-39.4)$ & 68 & $33.5(27.3-40.2)$ & \\
\hline Commuting-related physical activity & & & & & 0.058 \\
\hline Inactive/sedentary & 179 & $42.3(37.7-47.1)$ & 105 & $51.7(44.9-58.6)$ & \\
\hline Insufficiently active & 131 & $31.0(26.7-35.5)$ & 58 & $28.6(22.7-35.1)$ & \\
\hline Active & 113 & $26.7(22.7-31.1)$ & 40 & $19.7(11.7-25.6)$ & \\
\hline Leisure-time physical activity & & & & & 0.140 \\
\hline Inactive/sedentary & 292 & $69.0(64.5-73.3)$ & 155 & $76.4(70.1-81.8)$ & \\
\hline Insufficiently active & 56 & $13.2(10.3-16.7)$ & 18 & $8.9(5.5-13.4)$ & \\
\hline Active & 75 & $17.7(14.3-21.6)$ & 30 & $14.8(10.4-20.2)$ & \\
\hline
\end{tabular}

$95 \% \mathrm{Cl}=95 \%$ confidence interval. 
We found lower odds of obesity among individuals with lifetime histories of major depression. The results in the medical literature regarding this association are divergent, with positive results, ${ }^{35,36}$ negative results ${ }^{37-39}$ and inverse associations. ${ }^{40} \mathrm{~A}$ systematic review by Luppino et al. including 15 studies and 58,745 individuals found higher odds of major depression among individuals with obesity (OR 1.57; 95\% CI 1.23-2.01) and also reported that individuals with major depression had higher odds of obesity (OR 1.40; 95\% CI 1.15-1.71), although no association with overweight was found (OR 0.98; 95\% CI 0.83-1.16). ${ }^{35}$ However, Luppino et al. pointed out that differences in study populations might explain the heterogeneous results and suggested that some American studies had a positive association, whereas European studies did not show any significant associations between depression and obesity. ${ }^{35}$ An Asian study also reported an association of depression with underweight among men. ${ }^{40}$ A Brazilian cohort in Pelotas (Rio Grande do Sul) found that individuals with major depressive disorder had higher odds of obesity (OR 1.9; 95\% CI 1.09-3.46). ${ }^{41}$ The Brazilian National Health Survey (Pesquisa Nacional de Saúde, PNS 2013) also found a bidirectional association between depression and obesity. ${ }^{42}$ Paulitsch et al. found that the association of depression and obesity was mediated by weight perception, i.e. believing oneself to be fat. They also found that some groups were more vulnerable: being non-single, having more schooling and not engaging in physical activity. ${ }^{43}$

Our data contrasted with different Brazilian studies showing regional heterogeneity. In our study, the association between higher rates of commuting-related physical activity and lifetime history of MDD lost its significance after adjustment. Because our sample had a higher number of women than of men and commuting physical activity occurs more frequently among men than among women, ${ }^{44,45}$ our results probably lost significance after adjustment for sex.

Our study had some methodological strengthens. Trained psychiatrists made the psychiatric diagnoses of all participants, using a gold-standard instrument (SCID-I). The cardiovascular risk factors were evaluated using medical history and using clinical and laboratory assessments from onsite evaluation made by trained physicians.

The results from the study highlight the importance of diagnosing and treating depression within primary and secondary care. ${ }^{46}$

Table 2. Logistic regression models of the association between cardiovascular risk factors and lifetime history of major depression (crude and age and sex-adjusted)

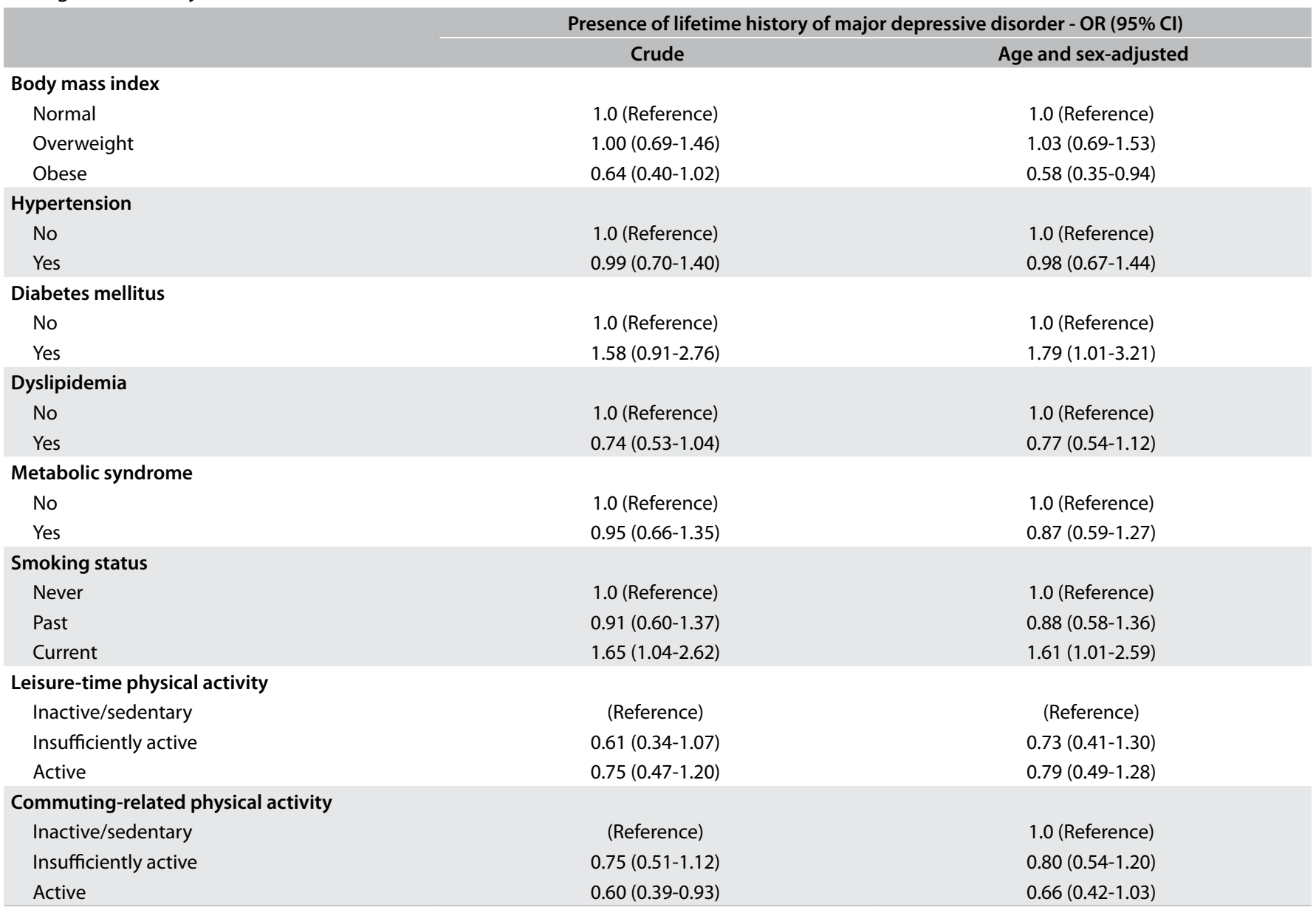

OR = odds ratio; $95 \% \mathrm{Cl}=95 \%$ confidence interval. 
Depression is an important risk factor for noncompliance with treatment of chronic diseases. Therefore, for effective control of cardiovascular risk factors, it is mandatory to recognize and treat depression. Depressive individuals are less active, with higher frequency of smoking and less adherence to prescribed medication. ${ }^{47}$ In addition, practicing physical activity can also be part of depression treatment. On the other hand, the effect of treating depression can have some impact regarding weight gain, which has been shown to have an important relationship with depression and cardiovascular risk factors and chronic diseases. Healthcare professionals can manage treatment of depression using better cardiovascular-profile drugs or considering other treatment options, such as psychotherapy, whenever possible. ${ }^{48}$

Our study also had some limitations, given that the cross-sectional study design did not permit evaluation of causality. Moreover, the diagnosis of diabetes was made based on only one measurement of fasting plasma glucose, as a consequence of budget limitations and logistic limitations on performing some laboratory tests such as hemoglobin Alc. Another important point is that our sample was young, with a mean age of around 40 years. The prevalence of cardiovascular risk factors increases with age, which may explain the lower frequency in our sample. In addition, although the sample in the first phase of the study with home interviews was representative of the population living in the metropolitan region of São Paulo, only a subsample of participants was invited in the second phase, and many of them refused to participate, thus introducing some selection bias. However, even considering the young age, we reported that there was higher prevalence of diabetes and smoking among the depressive participants than among the nondepressed participants.

\section{CONCLUSIONS}

In this sample of individuals living in the metropolitan region of São Paulo, the biggest city in Brazil, we found that individuals with lifetime major depression had higher odds of current tobacco use and of presenting diabetes mellitus. They also had lower odds of being obese, compared with nondepressed individuals. Healthcare professionals need to increase their awareness of the diagnosis of cardiovascular risk factors among individuals with major depression, in order to enhance preventive strategies and thus reduce morbidity and mortality in this high-risk group.

\section{REFERENCES}

1. GBD 2017 Causes of Death Collaborators. Global, regional, and national age-sex-specific mortality for 282 causes of death in 195 countries and territories, 1980-2017: a systematic analysis for the Global Burden of Disease Study 2017. Lancet. 2018;392(10159):1736-88. PMID: 30496103; https://doi.org/10.1016/S0140-6736(18)32203-7.

2. Appleton KM, Woodside JV, Arveiler D, et al. Depression and mortality: artifact of measurement and analysis? J Affect Disorders. 2013;151(2):63238. PMID: 23948631; https://doi.org/10.1016/j.jad.2013.07.010.
3. Chang CK, Hayes RD, Broadbent M, et al. All-cause mortality among people with serious mental illness (SMI), substance use disorders, and depressive disorders in southeast London: a cohort study. BMC Psychiatry. 2010;10:77. PMID: 20920287; https://doi.org/10.1186/1471-244X-10-77.

4. Wulsin $L R$, Singal BM. Do depressive symptoms increase the risk for the onset of coronary disease? A systematic quantitative review. Psychosom Med. 2003;65(2):201-10. PMID: 12651987; https://doi.org/10.1097/01. psy.0000058371.50240.e3.

5. Nicholson A, Kuper H, Hemingway H. Depression as an aetiologic and prognostic factor in coronary heart disease: a meta-analysis of 6,362 events among 146,538 participants in 54 observational studies. Eur Heart J. 2006;27(23):2763-74. PMID: 17082208; https://doi.org/10.1093/ eurheartj/ehl338.

6. Lawrence D, Mitrou F, Zubrick SR. Smoking and mental illness: results from population surveys in Australia and the United States. BMC Public Health. 2009;9:285. PMID: 19664203; https://doi.org/10.1186/1471-2458-9-285.

7. Strine TW, Mokdad AH, Dube SR, et al. The association of depression and anxiety with obesity and unhealthy behaviors among communitydwelling US adults. Gen Hosp Psychiatry. 2008;30(2):127-37. PMID: 18291294; https://doi.org/10.1016/j.genhosppsych.2007.12.008.

8. Seo Y, Je Y. A comparative study on cardiovascular disease risk factors in Korean adults according to clinical depression status. Psychiatry Res. 2018;263:88-93. PMID: 29510344; https://doi.org/10.1016/j. psychres.2018.02.052.

9. Koponen H, Jokelainen J, Keinanen-Kiukaanniemi S, Kumpusalo E, Vanhala M. Metabolic syndrome predispose to depressive symptoms: a population-based 7-year follow-up study. J Clin Psychiatry. 2008;69(2):17882. PMID: 18232723; https://doi.org/10.4088/jcp.v69n0202.

10. Vancampfort $D$, Correll CU, Wampers $M$, et al. Metabolic syndrome and metabolic abnormalities in patients with major depressive disorder: a meta-analysis of prevalences and moderating variables. Psychol Med. 2014;44(10):2017-28. PMID: 24262678; https://doi.org/10.1017/ S0033291713002778.

11. Viana MC, Teixeira MG, Beraldi F, Bassani IS, Andrade LH. São Paulo Megacity Mental Health Survey - a population-based epidemiological study of psychiatric morbidity in the São Paulo Metropolitan Area: aims, design and field implementation. Rev Bras Psiquiatr. 2009;31 (4):375-86. PMID: 20098829; https://doi.org/10.1590/s1516-44462009000400016.

12. First MB, Spitzer RL, Gibbon M, Williams JBW. Structured Clinical Interview for DSM-IV-TR Axis I Disorders, Research Version, Patient Edition. (SCID-I/P). New York: Biometrics Research: New York State Psychiatric Institute; 2002.

13. Matsudo S, Araújo T, Matsudo V, et al. Questionário Internacional de Atividade Física (IPAQ): estudo de validade e reprodutibilidade no Brasil. Revista Brasileira de Atividade Física \& Saúde. 2001;6(2):5-18. https:// doi.org/10.12820/rbafs.v.6n2p5-18.

14. Chobanian AV, Bakris GL, Black HR, et al. Seventh Report of the Joint National Committee on Prevention, Detection, Evaluation, and Treatment of High Blood Pressure. Hypertension. 2003;42(6):1206-52. PMID: 14656957; https://doi.org/10.1161/01.HYP.0000107251.49515.c2. 
15. American Diabetes Association. Diagnosis and classification of diabetes mellitus. Diabetes Care. 2010;33 Suppl 1 (Suppl 1):S62-9. PMID: 20042775; https://doi.org/10.2337/dc10-S062.

16. National Cholesterol Education Program (NCEP) Expert Panel on Detection, Evaluation, and Treatment of High Blood Cholesterol in Adults (Adult Treatment Panel III). Third Report of the National Cholesterol Education Program (NCEP) Expert Panel on Detection, Evaluation, and Treatment of High Blood Cholesterol in Adults (Adult Treatment Panel III) Final Report 2002. Circulation. 2002;106(25):3143-421. PMID: 12485966.

17. Centers for Disease Control and Prevention (CDC). National Center for Health Statistics (NCHS). National Health and Nutrition Examination Survey Data. Anthropometry procedures manual. Hyattsville, MD: U.S. Department of Health and Human Services, Centers for Disease Control and Prevention; 2004. Available from: https://www.cdc.gov/nchs/data/ nhanes/nhanes_07_08/manual_an.pdf. Accessed in 2020 (Mar 20).

18. Physical status: the use and interpretation of anthropometry. Report of a WHO Expert Committee. World Health Organ Tech Rep Ser. 1995;854:1452. PMID: 8594834.

19. Lin EH, Von Korff M, Alonso J, et al. Mental disorders among persons with diabetes - results from the World Mental Health Surveys. J Psychosom Res. 2008;65(6):571-80. PMID: 19027447; https://doi.org/10.1016/j. jpsychores.2008.06.007.

20. Mendenhall E, Norris SA, Shidhaye R et. al. Depression and Type 2 Diabetes in Low- and Middle-Income Countries: A Systematic Review. Diabetes Res Clin Pract. 2014;103(2):276-85. PMID: 24485858; https:// doi.org/10.1016/j.diabres.2014.01.001.

21. Roy T, Lloyd CE. Epidemiology of depression and diabetes: A systematic review. J Affect Dis. 2012;142S1:S8-21. PMID: 23062861; https://doi. org/10.1016/S0165-0327(12)70004-6.

22. Knol MJ, Twisk JW, Beekman AT, et al. Depression as a risk factor for the onset of type 2 diabetes mellitus: a meta-analysis. Diabetologia. 2006;49(5):837-45. PMID: 16520921; https://doi.org/10.1007/s00125006-0159-x.

23. MezukB, EatonWW, Albrecht S, Golden SH. Depression and type 2 diabetes over the lifespan: a meta-analysis. Diabetes Care. 2008 Dec;31(12):238390. PMID: 19033418; https://doi.org/10.2337/dc08-0985.

24. Mommersteeg PM, Herr R, Pouwer F, Holt RI, Loerbroks A. The association between diabetes and an episode of depressive symptoms in the 2002 World Health Survey: an analysis of 231,797 individuals from 47 countries. Diabet Med. 2013;30(6):e208-14. PMID: 23614792; https:// doi.org/10.1111/dme.12193.

25. Andersohn F, Schade R, Suissa S, Garbe E. Long-term use of antidepressants for depressive disorders and the risk of diabetes mellitus. Am J Psychiatry. 2009;166(5):591-8. PMID: 19339356; https:// doi.org/10.1176/appi.ajp.2008.08071065.

26. Timonen M, Rajala U, Jokelainen J, et al. Depressive symptoms and insulin resistance in young adult males: results from the Northern Finland 1966 birth cohort. Mol Psychiatry. 2006;11(10):929-33. PMID: 16702975; https://doi.org/10.1038/sj.mp.4001838.
27. Weaver LJ, Mendenhall E. Applying syndemics and chronicity: interpretations from studies of poverty, depression, and diabetes. Med Anthropol. 2014;33(2):92-108. PMID: 24512380; https://doi.org/ 10.1080/01459740.2013.808637.

28. Mendenhall E. Beyond Comorbidity: A Critical Perspective of Syndemic Depression and Diabetes in Cross-cultural Contexts. Med Anthropol Q. 2016;30(4):462-478. PMID: 25865829; https://doi.org/10.1111/maq.12215.

29. Mendenhall E, Kohrt BA, Norris SA, Ndetei D, Prabhakaran D. Noncommunicable disease syndemics: poverty, depression, and diabetes among low-income populations. Lancet. 2017;389(10072):951-963. PMID: 28271846; https://doi.org/10.1016/S0140-6736(17)30402-6.

30. Moulton CD, Pickup JC, Ismail K. The link between depression and diabetes: the search for shared mechanisms. Lancet Diabetes Endocrinol. 2015;3(6):46171. PMID: 25995124; https://doi.org/10.1016/S2213-8587(15)00134-5.

31. Bonnet F, Irving K, Terra JL, et al. Anxiety and depression are associated with unhealthy lifestyle in patients at risk of cardiovascular disease. Atherosclerosis. 2005;178(2):339-44. PMID: 15694943; https://doi. org/10.1016/j.atherosclerosis.2004.08.035.

32. Goodwin RD, Wall MM, Garey L, et al. Depression among current, former, and never smokers from 2005 to 2013: The hidden role of disparities in depression in the ongoing tobacco epidemic. Drug Alcohol Depend. 2017;173:191-9. PMID: 28209289; https://doi.org/10.1016/j.drugalcdep.2016.11.038.

33. Borçoi AR, Mendes SO, Gasparini Dos Santos J, et al. Risk factors for depression in adults: NR3C1 DNA methylation and lifestyle association. J Psychiatr Res. 2020;121:24-30. PMID:31731185; https://doi.org/10.1016/j. jpsychires.2019.10.011.

34. Rocha SAV, Hoepers ATC, Fröde TS, et al. Prevalence of smoking and reasons for continuing to smoke: a population-based study. J Bras Pneumol. 2019;45(4):e20170080. PMID: 30942283; https://doi. org/10.1590/1806-3713/e20170080

35. Luppino FS, de Wit LM, Bouvy PF, et al. Overweight, obesity, and depression: a systematic review and meta-analysis of longitudinal studies. Arch Gen Psychiatry. 2010;67(3):220-9. PMID: 20194822; https:// doi.org/10.1001/archgenpsychiatry.2010.2.

36. Simon GE, Von Korff M, Saunders K, et al. Association between obesity and psychiatric disorders in the US adult population. Arch Gen Psychiatry. 2006;63(7):824-30.PMID: 16818872; https://doi.org/10.1001/archpsyc.63.7.824.

37. Wiltink J, Beutel ME, Till Y, et al. Prevalence of distress, comorbid conditions and well-being in the general population. J Affect Disord. 2011;130(3):42937. PMID: 21106250; https://doi.org/10.1016/j.jad.2010.10.041

38. Jacka FN, Pasco JA, McConnell S, et al. Self-reported depression and cardiovascular risk factors in a community sample of women. Psychosomatics. 2007;48(1):54-9. PMID: 17209150; https://doi. org/10.1176/appi.psy.48.1.54

39. Konttinen H, Silventoinen K, Sarlio-Lähteenkorva S, Männistö S, Haukkala A. Emotional eating and physical activity self-efficacy as pathways in the association between depressive symptoms and adiposity indicators. Am J Clin Nutr. 2010;92(5):1031-9. PMID: 20861176; https://doi.org/10.3945/ ajen.2010.29732. 
40. Qin T, Liu W, Yin M, et al. Body mass index moderates the relationship between C-reactive protein and depressive symptoms: evidence from the China Health and Retirement Longitudinal Study. Sci Rep. 2017;7:39940 PMID: 28128231; https://doi.org/10.1038/srep39940.

41. Gomes AP, Soares ALG, Menezes AMB, et al. Adiposity, depression and anxiety: interrelationship and possible mediators. Rev Saude Publica. 2019;53:103. PMID: 31800914; https://doi.org/10.11606/S15188787.2019053001119.

42. Diderichsen F, Andersen I. The syndemics of diabetes and depression in Brazil - An epidemiological analysis. SSM Popul Health. 2018;7:002-2. PMID: 30581954; https://doi.org/10.1016/j.ssmph.2018.11.002.

43. Paulitsch RG, Demenech LM, Dumith SC. Association of depression and obesity is mediated by weight perception. J Health Psychol. 2020:1359105319897778. PMID: 31894712; https://doi. org/10.1177/1359105319897778.

44. Dinas PC, Koutedakis Y, Flouris AD. Effects of exercise and physical activity on depression. Ir J Med Sci. 2011;180(2):319-25. PMID: 21076975; https://doi.org/10.1007/s11845-010-0633-9.

45. Pitanga FJG, Matos SMA, Almeida MDC, Barreto SM, Aquino EML. Leisure-Time Physical Activity, but not Commuting Physical Activity, is Associated with Cardiovascular Risk among ELSA-Brasil Participants. Arq Bras Cardiol. 2018;1 10(1):36-43. PMID: 29412240; https://doi. org/10.5935/abc.20170178.

46. World Health Organization. Mental health in primary care: illusion or inclusion? World Health Organization 2018. Available from: https:// www.who.int/docs/default-source/primary-health-care-conference/ mental-health.pdf?sfvrsn=8c4621d2_2. Accessed in 2020 (Dec 18).

47. Bivanco-Lima D, Souza Santos Id, Vannucchi AM, Almeida Ribeiro MC. Cardiovascular risk in individuals with depression. Rev Assoc Med Bras (1992). 2013;59(3):298-304. PMID: 23684214; https://doi.org/10.1016/j. ramb.2012.12.006.

48. Cuijpers P, Quero S, Dowrick C, Arroll B. Psychological Treatment of Depression in Primary Care: Recent Developments. Curr Psychiatry Rep. 2019;21(12):129. PMID: 31760505; https://doi.org/10.1007/s11920019-1117-x.

The work described in this paper was conducted at the Institute of Psychiatry, Faculdade de Medicina FMUSP, Universidade de Sao Paulo, Sao Paulo, SP, BR, as part of the Megacity Study

Authors' contributions: Bivanco-Lima D: data curation (equal), formal analysis (equal), investigation (equal), writing-original draft (lead) and writing-review and editing (equal); Santos IS: data curation (equal), formal analysis (equal), methodology (equal), supervision (equal) and writing-review and editing (equal); Wang YP: conceptualization (equal), methodology (lead), project administration (lead), supervision (equal) and writing-review and editing (supporting); Viana MC: conceptualization (equal), methodology (equal), project administration (equal) and writing-review and editing (equal); Andrade LHSG: conceptualization (lead), investigation (equal), project administration (lead), supervision (lead) and writing-review and editing (equal); Lotufo PA: formal analysis (equal), methodology (equal), project administration (equal) and writing-review and editing (equal); and Benseñor IJM: conceptualization (lead); data curation (equal); formal analysis (lead); methodology (lead); supervision (lead) and writing-review and editing (lead). All authors reviewed and approved the final version of the manuscript to be released

Sources of funding: The São Paulo Megacity Mental Health Survey was funded by the Fundação de Amparo à Pesquisa do Estado de São Paulo (São Paulo Research Foundation; FAPESP 03/00204-3), and Fundo de Apoio à Ciência e Tecnologia de Vitória (FACITEC, Vitória [Municipal] Fund for the Support of Science and Technology; 002/2003 for instrument development). The Conselho Nacional de Desenvolvimento Científico e Tecnológico (Brazilian National Council for Scientific and Technological Development; CNPq 307933/2019-9) supported Dr. L. H. Andrade. The São Paulo Megacity Mental Health Survey was conducted in conjunction with the World Health Organization World Mental Health Survey Initiative. The main coordination center activities at Harvard University were supported by the United States National Institutes of Mental Health (R01-MH070884), John D. and Catherine T. MacArthur Foundation, Pfizer Foundation and United States Public Health Service (R13-MH066849, R01-MH069864 and R01-DA016558), as well as by the Fogarty International Center (FIRCA R03-TW006481), Pan-American Health Organization, Eli Lilly and Company Foundation, Ortho-McNeil Pharmaceutical, GlaxoSmithKline, Bristol-Myers Squibb and Shire. A complete list of the World Mental Health publications can be found at http://www.hcp.med.harvard.edu/wmh/. None of the sponsors had any role in the design, analysis, interpretation of results or preparation of this paper

\section{Conflict of interest: None}

Date of first submission: August 8, 2020

Last received: February 3, 2021

Accepted: February 18, 2021

\author{
Address for correspondence: \\ Danielle Bivanco-Lima \\ R. Doutor Cesário Mota Junior, 61 — 5 andar — Sala 03 \\ São Paulo (SP) — Brasil \\ CEP 01221-020 \\ Tel. (+55 11) 3367-7770 \\ E-mail: danielle.bivanco@gmail.com
}

